\title{
Clinical Outcomes of N1b Papillary Thyroid Cancer Patients Treated with Two Different Doses of Radioiodine Ablation Therapy
}

\author{
Meihua Jin ${ }^{1}$, Jonghwa Ahn ${ }^{1}$, Yu-Mi Lee ${ }^{2}$, Tae-Yon Sung ${ }^{2}$, Won Gu Kim¹, Tae Yong Kim, Jin-Sook Ryu \\ Won Bae Kim ${ }^{1}$, Young Kee Shong ${ }^{1}$, Min Ji Jeon ${ }^{1}$ \\ Departments of ${ }^{1}$ Internal Medicine, ${ }^{2}$ Surgery, ${ }^{3}$ Nuclear Medicine, Asan Medical Center, University of Ulsan College of \\ Medicine, Seoul, Korea
}

Background: The optimal dose of radioactive iodine (RAI) therapy for N1b papillary thyroid carcinoma (PTC) is controversial. We evaluated the clinical outcome of N1b PTC patients treated with either 100 or $150 \mathrm{mCi}$ of RAI.

Methods: We retrospectively analyzed N1b PTC patients who underwent total thyroidectomy and postoperative RAI therapy at a tertiary referral center between 2012 and 2017. As the baseline characteristics differed between treatment groups, we performed exact matching for various pathological factors according to RAI dose. We evaluated the response to therapy and recurrence-free survival (RFS) in the matched patients. Structural recurrent/persistent disease was defined as new structural disease detected after initial therapy, which was confirmed by cytology or pathology.

Results: Of the total 436 patients, $37(8.5 \%)$ received $100 \mathrm{mCi}$ of RAI and $399(91.5 \%)$ received $150 \mathrm{mCi}$ of RAI. After an exact 1:3 matching, 34 patients in the $100 \mathrm{mCi}$ group and 100 patients in the $150 \mathrm{mCi}$ group remained. There was no significant difference in response to therapy between the groups in the matched population $(P=0.63)$. An excellent response was achieved in $70.6 \%(n=24)$ of patients in the $100 \mathrm{mCi}$ group and $76.0 \%(n=76)$ in the $150 \mathrm{mCi}$ group. Two $(5.9 \%)$ patients in the $100 \mathrm{mCi}$ group and four (4.0\%) in the $150 \mathrm{mCi}$ group had recurrence and there was no significant difference in RFS between the groups in the matched population $(P=0.351)$.

Conclusion: There were no differences in response to therapy and RFS in N1b PTC patients according to RAI dose.

Keywords: Thyroid neoplasms; Neoplasm metastasis; Recurrence

\section{INTRODUCTION}

Postoperative radioactive iodine (RAI) therapy has been used for patients with differentiated thyroid carcinoma (DTC) to remove residual normal thyroid tissue after thyroidectomy or to treat potential metastatic disease $[1,2]$. DTC patients presenting

Received: 22 June 2020, Revised: 4 August 2020, Accepted: 6 August 2020 Corresponding author: Min Ji Jeon

Division of Endocrinology and Metabolism, Department of Internal Medicine, Asan Medical Center, University of Ulsan College of Medicine, 88 Olympic-ro 43-gil, Songpa-gu, Seoul 05505, Korea

Tel: +82-2-3010-1317, Fax: +82-2-3010-6962, E-mail: mj080332@gmail.com with lymph node (LN) metastasis have higher risks of distant metastasis and locoregional recurrence and lower remission rates compared with intrathyroidal tumors without clinical LN involvement [3]. Patients with LN metastasis were categorized as intermediate-risk group in the 2015 American Thyroid Association (ATA) guideline [1]. Furthermore, disease-specific mor-

\footnotetext{
Copyright (C) 2020 Korean Endocrine Society

This is an Open Access article distributed under the terms of the Creative Commons Attribution Non-Commercial License (https://creativecommons.org/ licenses/by-nc/4.0/) which permits unrestricted non-commercial use, distribution, and reproduction in any medium, provided the original work is properly cited.
} 
tality is higher in patients presenting with clinically significant LN metastasis [4-6].

The presence of lateral neck LN metastasis (N1b) is associated with a high rate of recurrence in papillary thyroid carcinoma (PTC) [7-9]. Furthermore, PTC patients with N1b disease showed inferior responses to surgery and RAI therapy compared to patients with only central neck LN metastasis (N1a) [10]. Thus, a high dose of RAI ablation/adjuvant therapy is routinely administered in PTC patients with N1b disease [11]. Recommendations for these patients often range from 100 to 250 $\mathrm{mCi}$ of RAI depending on the clinical situation, and most physicians treat $\mathrm{N} 1 \mathrm{~b}$ disease patients with $150 \mathrm{mCi}$ of RAI $[3,11]$. However, the optimal dose of RAI therapy remains controversial and there is no sufficient evidence that a higher dose of RAI therapy is associated with improvement in clinical outcomes for patients with $\mathrm{N} 1 \mathrm{~b}$ disease. A recent study reported that administering more than $100 \mathrm{mCi}$ of $\mathrm{RAI}$ as adjuvant therapy in $\mathrm{N} 1 \mathrm{~b}$ disease is unlikely to improve the initial response to therapy, especially in young patients [3].

Therefore, the aim of this study was to evaluate the clinical outcome of patients with N1b disease who were treated with either 100 or $150 \mathrm{mCi}$ of RAI.

\section{METHODS}

\section{Patients}

This study was a retrospective analysis of N1b PTC patients who underwent total thyroidectomy and postoperative RAI therapy at the Asan Medical Center (Seoul, South Korea) between 2012 and 2017. We included adult PTC patients with N1b disease confirmed at final pathology and who underwent either 100 or $150 \mathrm{mCi}$ of RAI. Exclusion criteria were as follows: patients with distant metastasis initially; pathological diagnosis of follicular, medullary, poorly DTC, or anaplastic thyroid cancer; and patients without adequate follow-up data. Finally, 436 patients were included for analysis (Fig. 1). The study protocol was approved by the Institutional Review Board of the Asan Medical Center (study number: 2019-0676). Written informed consent by the patients was waived due to a retrospective nature of our study.

\section{Treatment and follow-up protocol}

All patients underwent total thyroidectomy with central and lateral neck dissection. For RAI therapy, patients received ablative doses of ${ }^{131}$ I after thyroxine withdrawal or after two consecutive injections of recombinant human thyrotropin alpha (rhTSH). A post-therapy whole body scan (WBS) was obtained 5 to 7 days after administration of ${ }^{131} \mathrm{I}$. After RAI therapy, patients were regularly followed up with levothyroxine supplementation. Physical examinations, including neck palpation, thyroid function test, serum thyroglobulin (Tg), and anti-Tg antibody $(\mathrm{TgAb})$ measurement, were done in all patients at each visit. To assess the response to therapy, a diagnostic WBS, stimulated Tg (sTg) with $\mathrm{TgAb}$ measurement, and neck ultrasonography (US)

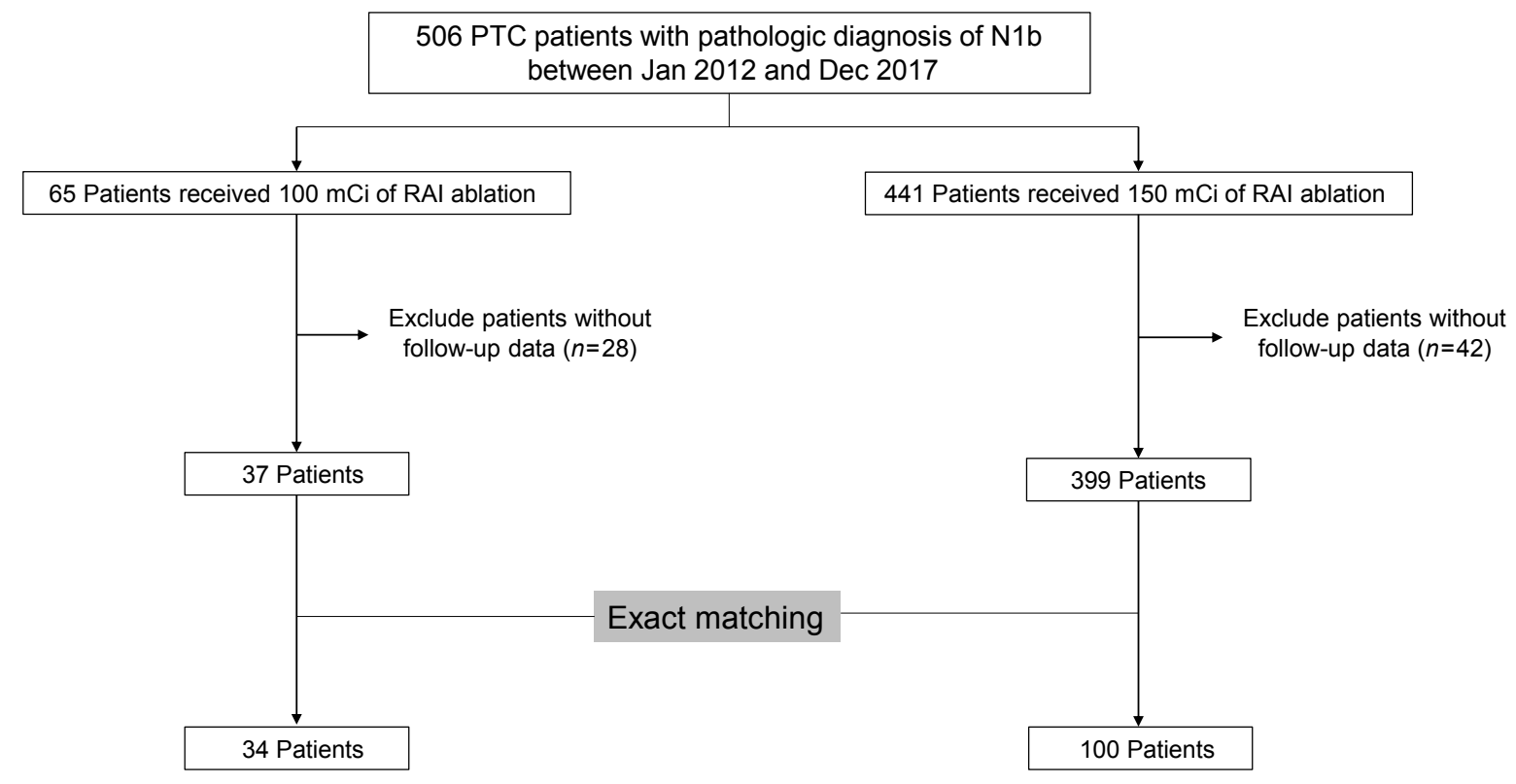

Fig. 1. Flow chart of patient enrollment. PTC, papillary thyroid carcinoma; RAI, radioactive iodine. 
were done 6 to 12 months after RAI therapy [12-14]. The level of sTg was determined after thyroxine withdrawal or injection of rhTSH. After the response to therapy was assessed, neck US was performed every 12 to 24 months. If there were abnormal findings on neck US, US-guided fine-needle aspiration cytology was done. Other imaging studies, including neck/chest computed tomography or ${ }^{18} \mathrm{~F}$-fluorodeoxyglucose-positron emission tomography, were performed when needed.

\section{Definition}

The risk of recurrence was determined by the ATA risk stratification system $[1,15]$. Intermediate-risk of recurrence was defined as microscopic tumor invasion into perithyroidal soft tissue, RAI-avid metastatic foci in the neck on the first posttreatment whole body RAI scan, aggressive histology, PTC with vascular invasion, clinical $\mathrm{N} 1$ or $>5$ pathologic N1 with all involved LNs $<3 \mathrm{~cm}$ in largest dimension, and multifocal papillary microcarcinoma with extrathyroidal extension (ETE) and $B R A F^{\mathrm{V} 600 \mathrm{E}}$ mutated if known. High-risk of recurrence was defined as the macroscopic invasion of the tumor into the perithyroidal soft tissue (gross ETE), incomplete tumor resection, distant metastasis, postoperative serum Tg suggestive of distant metastasis, pathological N1 with any metastatic LNs $\geq 3 \mathrm{~cm}$ in the largest dimension or with extranodal extension (ENE) $[1,15]$.

The response to initial therapy was classified according to the 2015 ATA management guidelines [1]: (1) excellent response if patients achieved $\mathrm{sTg}<1 \mathrm{ng} / \mathrm{mL}$ with negative $\mathrm{TgAb}$ and had no abnormal finding in neck US and diagnostic WBS; (2) indeterminate response if patients achieved sTg between 1 to $10 \mathrm{ng} /$ $\mathrm{mL}$ or sTg was stable or declining in the absence of structural disease; (3) biochemical incomplete response if patients had sTg $>10 \mathrm{ng} / \mathrm{mL}$ or rising TgAb levels in the absence of structural disease; or (4) structural incomplete response if patients had abnormal findings on imaging.

Structural recurrent/persistent disease was defined as the detection of new structural disease after initial therapy, which was confirmed by cytology or pathology. Recurrence-free survival (RFS) was defined as the time from total thyroidectomy to the detection of structural recurrent/persistent disease or the end of follow-up.

\section{Statistical analysis}

Statistical analyses were conducted using R version 3.5.1 (R Foundation for Statistical Computing, Vienna, Austria; http:// www.R.project.org) and survival curves were drawn using GraphPad Prism version 5.0 (GraphPad Software, San Diego,
CA, USA; http://www.graphpad.com). Continuous variables are presented as mean \pm standard deviation, and categorical variables are presented as number (percentage). We used the $t$ test to compare continuous variables and the chi-square test to compare categorical variables. Survival curves were plotted using the Kaplan-Meier method, and the log-rank test was used to determine significance. We performed exact matching of patients according to RAI dose for further analysis. An exact 1:3 matching was performed for factors, the number and size of LNs, presence of ETE, and presence of ENE. LN numbers were matched using \pm 4 as an acceptable boundary, and the sizes of LNs were matched using $\pm 1 \mathrm{~cm}$ as an acceptable boundary. The presence of ETE and ENE was exactly matched in both treatment groups. Differences were considered statistically significant at $P<0.05$.

\section{RESULTS}

\section{Baseline characteristics of all patients}

Among the 436 patients included in this study, 37 (8.5\%) received $100 \mathrm{mCi}$ of RAI and $399(91.5 \%)$ received $150 \mathrm{mCi}$ of RAI. Baseline characteristics of these 436 patients are listed in Table 1 . The mean age of the patients was 43.8 years and 272 patients $(62 \%)$ were female. The mean tumor size was $2.1 \mathrm{~cm}$ and most had ETE: 24\% with microscopic ETE and 56\% with gross ETE. The mean number and size of metastatic LNs was 13.6 and $1.3 \mathrm{~cm}$, respectively, and 57\% showed ENE. Patients who received $150 \mathrm{mCi}$ of RAI had more ETE $(P=0.005)$, larger metastatic LNs $(P<0.001)$, and more frequent $\mathrm{ENE}(P<0.001)$. There were more patients with T3 and T4 disease in the 150 $\mathrm{mCi}$ group $(P=0.001)$, but there was no significant difference in TNM stage according to RAI dose. Among the total patients, 95 $(21.8 \%)$ patients were classified as the intermediate-risk group by the ATA risk stratification, and 341 (78.2\%) were classified as the high-risk group. There were more patients with a highrisk of recurrence in the $150 \mathrm{mCi}$ group $(P<0.001)$. In terms of surgical extent, 347 (79.6\%) were underwent unilateral modified radical neck dissection (MRND) and others underwent bilateral MRND and there was no significant difference in surgical extent between two treatment groups $(P=0.65)$. There was no significant difference between the extent of lateral LN metastasis in two groups $(P=0.87)$. Five patients $(1.1 \%)$ had residual tumor after surgery. All of the patients who received 150 $\mathrm{mCi}$ of RAI were prepared with levothyroxine withdrawal and $30(81.1 \%)$ patients who treated with $100 \mathrm{mCi}$ were prepared with rhTSH. At the time of RAI therapy, $15.9 \%$ of patients were 


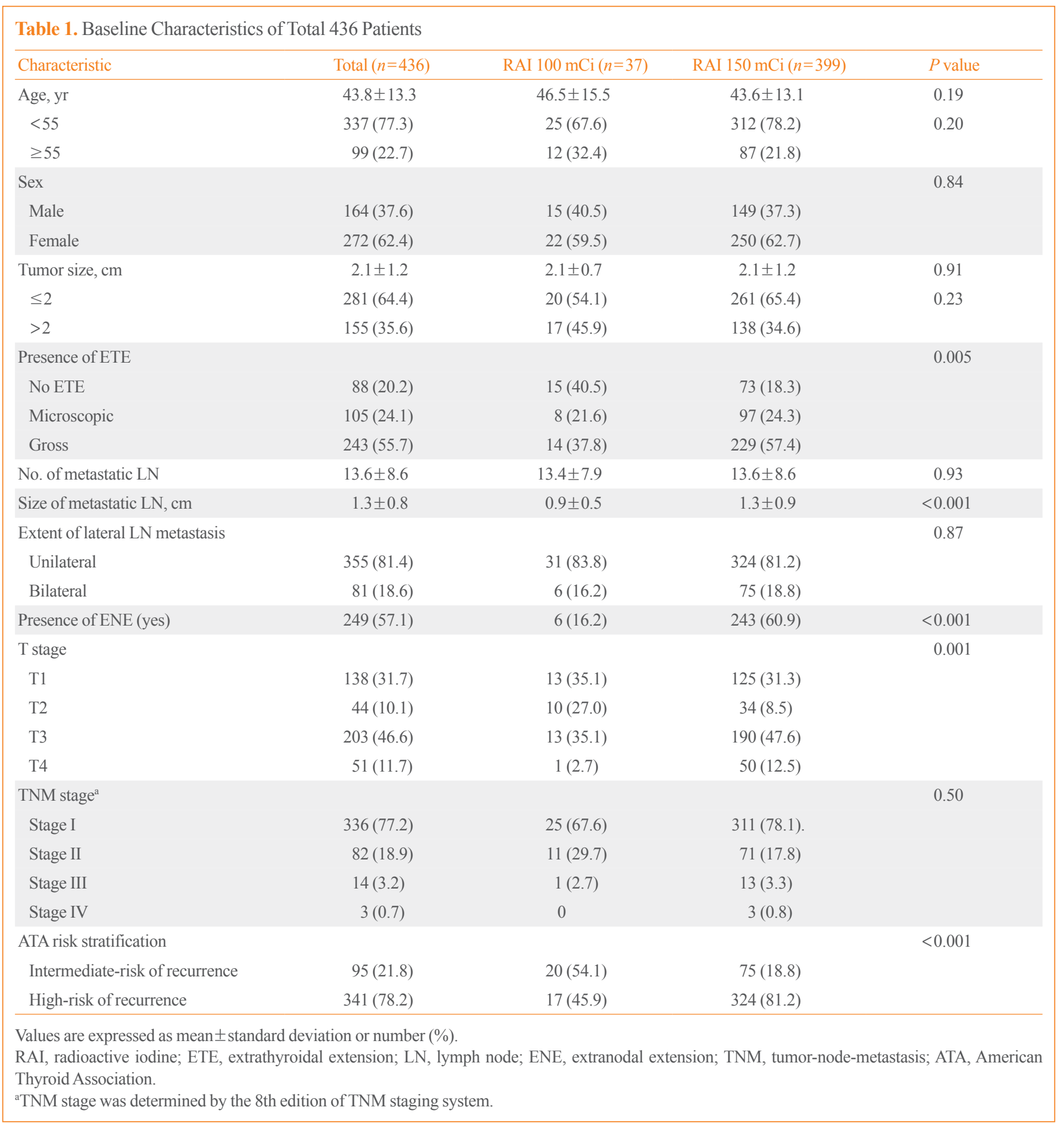

positive for $\operatorname{TgAb}$ (reference $<60 \mathrm{U} / \mathrm{mL}$ ) and the median $\mathrm{sTg}$ among patients with negative $\mathrm{TgAb}$ was $2.10 \mathrm{ng} / \mathrm{mL}$ (range, 0.08 to 1,790.00). The proportion of patients with positive $\mathrm{TgAb}$ and median sTg levels was not significantly different between two groups ( $P=0.99$ and $P=0.40$, respectively).

\section{Baseline characteristics of matched patients}

As the baseline characteristics were different between groups according to RAI dose, we performed exact matching for the pathologic characteristics as described in the methods. After matching, 34 patients in the $100 \mathrm{mCi}$ group and 100 patients in the $150 \mathrm{mCi}$ group remained (Fig. 1). The baseline characteris- 
tics of the matched 134 patients are shown in Table 2. After matching, the presence of ETE, number of LNs, size of LNs, and presence of ENE, which were all well-known risk factors for the recurrence of thyroid cancer, were not significantly different between the two groups. There was also no significant difference in T stage, ATA risk stratification and surgical extent between the two groups (Table 2).

\section{Response to therapy classification and structural recurrent/persistent disease}

The responses to initial therapy according to RAI dose in the total and matched populations are shown in Table 3 . There were no significant differences in the response to therapy classification between the treatment groups in either total or matched patients ( $P=0.37$ and $P=0.63$, respectively). In the matched population, $71 \%$ of patients $(n=24)$ in the $100 \mathrm{mCi}$ group and $76 \%$ $(n=76)$ in the $150 \mathrm{mCi}$ group achieved excellent response.

During a median follow-up period of 4.8 years (range, 0.5 to $8.1), 48(11.0 \%)$ of the total 436 patients had structural recurrent/persistent disease: three patients $(8.1 \%)$ in the $100 \mathrm{mCi}$ group and $45(11.3 \%)$ in the $150 \mathrm{mCi}$ group. Of total 48 patients who had structural recurrent/persistent disease, 41 patients $(9.4 \%)$ had locoregional metastasis, two patients $(0.5 \%)$ had distant metastasis, and four $(0.9 \%)$ had both locoregional and distant metastasis. The RFS curves are shown in Fig. 2A. RFS did not differ significantly according to RAI dose $(P=0.76)$. The results were consistent in the matched population. Among the 134 matched patients, six $(4.5 \%)$ had disease recurrence: two patients $(5.9 \%)$ in the $100 \mathrm{mCi}$ group and four $(4.0 \%)$ in the $150 \mathrm{mCi}$ group. The RFS curves of the matched patients are shown in Fig. 2B. There was no significant difference in RFS between the groups $(P=0.35)$.

\section{DISCUSSION}

This was a retrospective study evaluating two different doses of RAI in N1b PTC patients. In the current study, 48 (11.0\%) of the total 436 patients had structural recurrent/persistent disease after initial therapy, and the rate was similar with previous studies $[1,3,16]$. Because of differences in pathological characteristics between the groups, we evaluated the clinical outcomes of these patients after exact matching of various pathological risk factors. In both the total and matched cohorts, response to therapy classification and RFS was not different between 100 and $150 \mathrm{mCi}$ of RAI therapy.

Recent studies demonstrated that low-dose RAI is sufficient

\begin{tabular}{|c|c|c|c|}
\hline Characteristic & $\begin{array}{l}\text { RAI } 100 \mathrm{mCi} \\
\quad(n=34)\end{array}$ & $\begin{array}{l}\text { RAI } 150 \mathrm{mCi} \\
\quad(n=100)\end{array}$ & $P$ value \\
\hline Age, yr & $47.9 \pm 15.3$ & $45.5 \pm 12.7$ & 0.37 \\
\hline$<55$ & $22(64.7)$ & $76(76.0)$ & 0.29 \\
\hline$\geq 55$ & $12(35.3)$ & $24(24.0)$ & \\
\hline Sex & & & 0.43 \\
\hline Male & $13(38.2)$ & $29(29.0)$ & \\
\hline Female & $21(61.8)$ & $71(71.0)$ & \\
\hline Tumor size, $\mathrm{cm}$ & $2.1 \pm 0.7$ & $2.0 \pm 1.1$ & 0.59 \\
\hline$\leq 2$ & $17(50.0)$ & $62(62.0)$ & 0.30 \\
\hline$>2$ & $17(50.0)$ & $38(38.0)$ & \\
\hline Presence of ETE & & & 0.98 \\
\hline No ETE & $14(41.2)$ & $42(42.0)$ & \\
\hline Microscopic & $6(17.6)$ & $18(18.0)$ & \\
\hline Gross & $14(41.2)$ & $42(42.0)$ & \\
\hline No. of metastatic LN & $12.2 \pm 6.8$ & $10.9 \pm 5.6$ & 0.28 \\
\hline Size of metastatic LN, cm & $0.9 \pm 0.5$ & $0.9 \pm 0.5$ & 0.96 \\
\hline Extent of lateral LN metastasis & & & 0.79 \\
\hline Unilateral & $29(85.3)$ & $89(89.0)$ & \\
\hline Bilateral & $5(14.7)$ & $11(11.0)$ & \\
\hline Presence of ENE (yes) & $6(17.6)$ & $18(18.0)$ & 0.99 \\
\hline T stage & & & 0.31 \\
\hline $\mathrm{T} 1$ & $11(32.4)$ & $38(38.0)$ & \\
\hline $\mathrm{T} 2$ & $10(29.4)$ & $15(15.0)$ & \\
\hline $\mathrm{T} 3$ & $12(35.3)$ & $42(42.0)$ & \\
\hline $\mathrm{T} 4$ & $1(2.9)$ & $5(5.0)$ & \\
\hline TNM stage $^{b}$ & & & 0.52 \\
\hline Stage I & $22(64.7)$ & $76(76.0)$ & \\
\hline Stage II & $11(32.4)$ & $21(21.0)$ & \\
\hline Stage III & $1(2.9)$ & $2(2.0)$ & \\
\hline Stage IV & 0 & $1(1.0)$ & \\
\hline ATA risk stratification & & & 0.84 \\
\hline Intermediate-risk of recurrence & $18(52.9)$ & $49(49.0)$ & \\
\hline High-risk of recurrence & $16(47.1)$ & $51(51.0)$ & \\
\hline
\end{tabular}

Values are expressed as mean \pm standard deviation or number (\%). RAI, radioactive iodine; ETE, extrathyroidal extension; LN, lymph node; ENE, extranodal extension; TNM, tumor-node-metastasis; ATA, American Thyroid Association.

${ }^{a}$ An exact 1:3 matching was performed for the factors; the number and size of LN, the presence of ETE, and the presence of ENE; ${ }^{b} \mathrm{TNM}$ stage was determined by the 8 th edition of TNM staging system.

for remnant ablation for low- to intermediate-risk DTC patients, and many experts advocate the use of lower doses of RAI [2,1720]. In 2012, Schlumberger et al. [2] and Mallick et al. [17] 
Table 3. Response to Therapy Classification in Total and Matched Patients

\begin{tabular}{|c|c|c|c|c|c|c|}
\hline \multirow[b]{2}{*}{ Variable } & \multicolumn{3}{|c|}{ Total patients } & \multicolumn{3}{|c|}{ Matched patients } \\
\hline & $\begin{array}{c}\text { RAI } 100 \mathrm{mCi} \\
\quad(n=37)\end{array}$ & $\begin{array}{l}\text { RAI } 150 \mathrm{mCi} \\
\quad(n=399)\end{array}$ & $P$ value & $\begin{array}{c}\text { RAI } 100 \mathrm{mCi} \\
\quad(n=34)\end{array}$ & $\begin{array}{l}\text { RAI } 150 \mathrm{mCi} \\
\quad(n=100)\end{array}$ & $P$ value \\
\hline Response & & & 0.37 & & & 0.63 \\
\hline Excellent response & $26(70.3)$ & $249(62.4)$ & & $24(70.6)$ & $76(76.0)$ & \\
\hline Indeterminate & $4(10.8)$ & $61(15.3)$ & & $4(11.8)$ & $13(13.0)$ & \\
\hline Biochemical incomplete & 0 & $24(6.0)$ & & 0 & $1(1.0)$ & \\
\hline Structural incomplete & $7(18.9)$ & $65(16.3)$ & & $6(17.6)$ & $10(10.0)$ & \\
\hline
\end{tabular}

Values are expressed as number (\%). $P$ value for chi-square test.

$\mathrm{RAI}$, radioactive iodine.
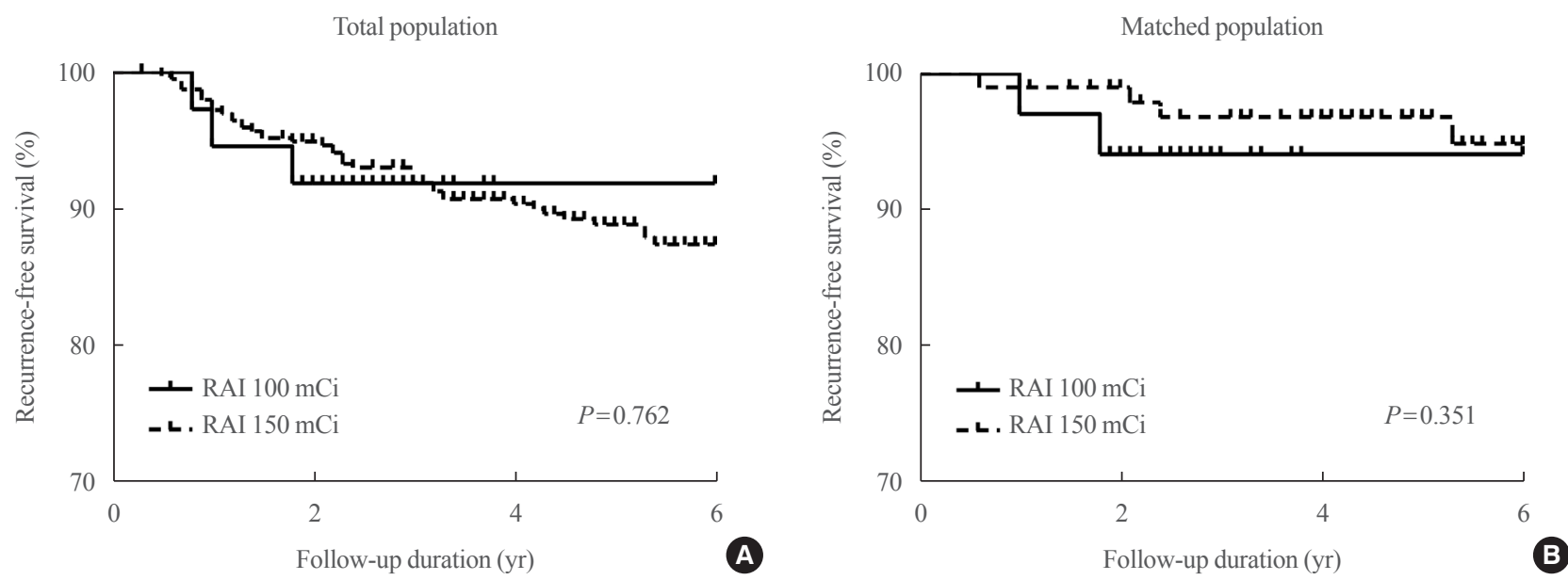

Fig. 2. Kaplan-Meier analysis of recurrence-free survival according to different doses of radioactive iodine (RAI) therapy. (A) Recurrencefree survival in total 436 patients. (B) Recurrence-free survival in the matched 134 patients according to different doses of RAI.

compared $1.1 \mathrm{GBq}(30 \mathrm{mCi})$ and $3.7 \mathrm{GBq}(100 \mathrm{mCi})$ in DTC patients and reported similar rates of effectiveness and fewer adverse events in the lower RAI group than in the higher RAI group. The presence of N1b is an important prognostic factor for DTC patients, and a high dose of RAI for adjuvant therapy might be helpful $[4,21]$. However, patients undergoing highdose RAI tend to have more adverse effects, such as sialadenitis, xerostomia, decreased gonadal function, and secondary neoplasm [22-25]. Considering the effectiveness and adverse effects, we need more accurate evidence for the efficacy of higher doses of RAI as a treatment option.

To our knowledge, there are no randomized controlled studies comparing the efficacy and safety of lower and higher doses of RAI in N1b PTC patients, and only a few retrospective studies have been reported $[3,16]$. A previous study involving 181 PTC patients with $\mathrm{N} 1 \mathrm{~b}$ disease who were treated with $100,150 \mathrm{mCi}$, or high activity (>200 mCi) RAI therapy demonstrated that ad- ministering more than $100 \mathrm{mCi}$ of RAI as adjuvant therapy is unlikely to improve the initial response to therapy, especially in young patients [3]. Another study involving 253 DTC patients with $\mathrm{T} 4$ or N1b disease demonstrated that adjuvant RAI therapy with rhTSH plus $3.7 \mathrm{GBq}(100 \mathrm{mCi})$ had a comparable ablation success rate and RFS compared to thyroid hormone withdrawal plus $5.5 \mathrm{GBq}(150 \mathrm{mCi})$ [16]. Our current study also showed similar results to previous studies. In the 134 matched patients with $\mathrm{N} 1 \mathrm{~b}$ disease in the current study, the response to therapy and RFS did not differ significantly between $100 \mathrm{mCi}$ and 150 mCi of RAI therapy. This suggests that $100 \mathrm{mCi}$ of RAI for N1b PTC patients is sufficient for remnant ablation. However, patients with more favorable pathological characteristics tended to remain after exact matching in our present study. The proportion of patients with ATA high-risk was $81.2 \%$ in $150 \mathrm{mCi}$ group, and it was decreased to $51.0 \%$ after matching. Consequently, the proportion of patients with structural recurrent/persistent 
disease in $150 \mathrm{mCi}$ group decreased from $11.3 \%$ to $4.0 \%$ after matching. Therefore, the result may be limited for the patients with more favorable pathologic findings and further studies for higher risk N1b disease are needed.

There were several limitations in the current study. First, it was a retrospectively designed study involving a small number of patients. Second, we did not investigate the adverse effects of different RAI administration strategies. Third, because the follow-up period was relatively short, there was no data about disease-specific or overall survival. Fourth, as we described above, the result of this study may be limited for the patients with more favorable pathologic findings. However, a strength of the current study is that it evaluated the effect of two different doses of $\mathrm{RAI}$ in N1b disease patients who were well-matched for tumor and LN factors.

In conclusion, there was no significant difference in response to therapy or RFS in PTC patients with N1b disease between 100 and $150 \mathrm{mCi}$ of RAI therapy after exact matching of various pathological factors. These findings may help clinicians determine the optimal dose of RAI therapy in patients with N1b PTC.

\section{CONFLICTS OF INTEREST}

No potential conflict of interest relevant to this article was reported.

\section{ACKNOWLEDGMENTS}

This study was supported by a grant (No. 2020IL0003) from the Asan Institute for Life Sciences, Asan Medical Center, Seoul, Korea.

\section{AUTHOR CONTRIBUTIONS}

Conception or design: M.J., J.A., Y.M.L., T.Y.S., W.G.K., T.Y.K., J.S.R., W.B.K., Y.K.S., M.J.J. Acquisition, analysis, or interpretation of data: M.J., J.A., Y.M.L., T.Y.S., W.G.K., T. Y.K., J.S.R., W.B.K., Y.K.S., M.J.J. Drafting the work or revising: M.J., M.J.J. Final approval of the manuscript: M.J., M.J.J.

\section{ORCID}

Meihua Jin https://orcid.org/0000-0003-0196-631X

Min Ji Jeon https://orcid.org/0000-0002-1297-107X

\section{REFERENCES}

1. Haugen BR, Alexander EK, Bible KC, Doherty GM, Mandel SJ, Nikiforov YE, et al. 2015 American Thyroid Association management guidelines for adult patients with thyroid nodules and differentiated thyroid cancer: the American Thyroid Association guidelines task force on thyroid nodules and differentiated thyroid cancer. Thyroid 2016;26:1-133.

2. Schlumberger M, Catargi B, Borget I, Deandreis D, Zerdoud $\mathrm{S}$, Bridji B, et al. Strategies of radioiodine ablation in patients with low-risk thyroid cancer. N Engl J Med 2012;366: 1663-73.

3. Sabra MM, Grewal RK, Ghossein RA, Tuttle RM. Higher administered activities of radioactive iodine are associated with less structural persistent response in older, but not younger, papillary thyroid cancer patients with lateral neck lymph node metastases. Thyroid 2014;24:1088-95.

4. Podnos YD, Smith D, Wagman LD, Ellenhorn JD. The implication of lymph node metastasis on survival in patients with well-differentiated thyroid cancer. Am Surg 2005;71:731-4.

5. Zaydfudim V, Feurer ID, Griffin MR, Phay JE. The impact of lymph node involvement on survival in patients with papillary and follicular thyroid carcinoma. Surgery 2008;144: 1070-7.

6. Leboulleux S, Rubino C, Baudin E, Caillou B, Hartl DM, Bidart JM, et al. Prognostic factors for persistent or recurrent disease of papillary thyroid carcinoma with neck lymph node metastases and/or tumor extension beyond the thyroid capsule at initial diagnosis. J Clin Endocrinol Metab 2005;90: 5723-9.

7. Ito Y, Kudo T, Takamura Y, Kobayashi K, Miya A, Miyauchi A. Lymph node recurrence in patients with N1b papillary thyroid carcinoma who underwent unilateral therapeutic modified radical neck dissection. World J Surg 2012;36:5937.

8. Baek SK, Jung KY, Kang SM, Kwon SY, Woo JS, Cho SH, et al. Clinical risk factors associated with cervical lymph node recurrence in papillary thyroid carcinoma. Thyroid 2010; 20:147-52.

9. Lee SH, Roh JL, Gong G, Cho KJ, Choi SH, Nam SY, et al. Risk factors for recurrence after treatment of N1b papillary thyroid carcinoma. Ann Surg 2019;269:966-71.

10. Liu YQ, Li H, Liu JR, Lin YS. Unfavorable responses to radioiodine therapy in N1b papillary thyroid cancer: a propensity score matching study. Endocr Pract 2019;25:1286-94.

11. Beierwaltes WH, Rabbani R, Dmuchowski C, Lloyd RV, 
Eyre P, Mallette S. An analysis of "ablation of thyroid remnants" with I-131 in 511 patients from 1947-1984: experience at University of Michigan. J Nucl Med 1984;25:1287-93.

12. Jeon MJ, Kim WG, Park WR, Han JM, Kim TY, Song DE, et al. Modified dynamic risk stratification for predicting recurrence using the response to initial therapy in patients with differentiated thyroid carcinoma. Eur J Endocrinol 2013;170: 23-30.

13. Kim WB. A closer look at papillary thyroid carcinoma. Endocrinol Metab (Seoul) 2015;30:1-6.

14. Yi KH. The revised 2016 Korean Thyroid Association guidelines for thyroid nodules and cancers: differences from the 2015 American Thyroid Association guidelines. Endocrinol Metab (Seoul) 2016;31:373-8.

15. Lamartina L, Grani G, Durante C, Borget I, Filetti S, Schlumberger M. Follow-up of differentiated thyroid cancer: what should (and what should not) be done. Nat Rev Endocrinol 2018;14:538-51.

16. Jeong SY, Lee SW, Kim WW, Jung JH, Lee WK, Ahn BC, et al. Clinical outcomes of patients with T4 or N1b well-differentiated thyroid cancer after different strategies of adjuvant radioiodine therapy. Sci Rep 2019;9:5570.

17. Mallick U, Harmer C, Yap B, Wadsley J, Clarke S, Moss L, et al. Ablation with low-dose radioiodine and thyrotropin alfa in thyroid cancer. N Engl J Med 2012;366:1674-85.

18. Han JM, Kim WG, Kim TY, Jeon MJ, Ryu JS, Song DE, et al. Effects of low-dose and high-dose postoperative radioiodine therapy on the clinical outcome in patients with small differentiated thyroid cancer having microscopic extrathyroidal extension. Thyroid 2014;24:820-5.
19. Caglar M, Bozkurt FM, Akca CK, Vargol SE, Bayraktar M, Ugur O, et al. Comparison of 800 and $3700 \mathrm{MBq}$ iodine-131 for the postoperative ablation of thyroid remnant in patients with low-risk differentiated thyroid cancer. Nucl Med Commun 2012;33:268-74.

20. Castagna MG, Cevenini G, Theodoropoulou A, Maino F, Memmo S, Claudia C, et al. Post-surgical thyroid ablation with low or high radioiodine activities results in similar outcomes in intermediate risk differentiated thyroid cancer patients. Eur J Endocrinol 2013;169:23-9.

21. Sellers M, Beenken S, Blankenship A, Soong SJ, TurbatHerrera E, Urist M, et al. Prognostic significance of cervical lymph node metastases in differentiated thyroid cancer. Am J Surg 1992;164:578-81.

22. Rosario PW, Borges MA, Purisch S. Preparation with recombinant human thyroid-stimulating hormone for thyroid remnant ablation with $131 \mathrm{I}$ is associated with lowered radiotoxicity. J Nucl Med 2008;49:1776-82.

23. Kloos RT, Duvuuri V, Jhiang SM, Cahill KV, Foster JA, Burns JA. Nasolacrimal drainage system obstruction from radioactive iodine therapy for thyroid carcinoma. J Clin Endocrinol Metab 2002;87:5817-20.

24. Jeong SY, Kim HW, Lee SW, Ahn BC, Lee J. Salivary gland function 5 years after radioactive iodine ablation in patients with differentiated thyroid cancer: direct comparison of preand postablation scintigraphies and their relation to xerostomia symptoms. Thyroid 2013;23:609-16.

25. Mandel SJ, Mandel L. Radioactive iodine and the salivary glands. Thyroid 2003;13:265-71. 